\title{
ĐÁNH GIÁ RỐI LOẠN GIỌNG CỦA BÊNNH NHÂN SAU CẮT THANH QUẢN BÁN PHẦN
}

\author{
Đàm Thu Hiền', Phạm Thị Bích Đào ${ }^{1,2}$, \\ Trần Văn Tâm², Mai Thị Mai Phương ${ }^{3}$
}

\section{TÓM TẮT}

Phẫu thuật cắt thanh quản bán phânchiếm 31,2\% tổng số các ca phẫu thuật ung thư thanh quản. Việc cắt bỏ một phần cấu trúc thanh quản sẽ làm thay đổi giọng. Chúng tôi tiến hành nghiên cứu "Đánh giá rối loạn giọng của bệnh nhân sau cắt thanh quản bán phần" trên 30 bênh nhân được cắt thanh quản bán phần tại Bệnh viện Tai Mũi Họng trung ương, được phân tích gionng trước và sau cắt thanh quản bán phẩn bằng chương trình phân tích âm PRAAT. Kết quả: Tuổi: 45-65 tuổi: $63.33 \%$, trung bình 60.16, Nam $100 \%$. Lý do vào viện: khàn tiếng: $100 \%$. Đánh giá giọng trước cắt thanh quản bán phần (TQBP): Phát âm nguyên âm: âm trung tính (chỉ số Shimmer 5.673, Jitter 2.005\%, HNR 26.783), tần số âm cơ bản: $128 \pm$ 2.8; biên đô âm cơ bản: $36 \pm 2.1$, trường đô âm cơ bản: $89 \pm$ 3.7. Thanh điệu: Thanh ngang: F0, F0-30, F0-50, F0-60, thanh huyền: $F 0, F 0-30, F 0-50, F 0-80$, thanh sắc: $F 0-60, F 0-80, F 0+70$, thanh hỏi: $F 0-F 0-$ 85, F0-90, F0-F0+30, thanh ngã: $F 0, F 0-60, F 0+40$, F0+90, thanh nă̆ng: F0, F0-90, F0-120, F0-140. Sau cắt TQBP: âm trüng tính (chỉ số Shimmer 5.874, Jitter 2.452, HNR 28.459; tần số âm cơ bản: $98 \pm 3.7$; biên đô âm cơ bản: $47 \pm 4.3$, trường đô âm cơ bản: $119 \pm$ 5.6. Thanh điệ: Thanh ngang: F0, F0-50, F0-70, F080, thanh huyền: $F 0, F 0-55, F 0-76, F 0-90$, thanh sắc: F0- 70, F0-90, F0+60, thanh hỏi: F0, F0-95, F0-105, F0-120, F0-90, F0-80, F0+30, thanh ngã: F0, F0-70, $\mathrm{F0}+30$, F0+50,thanh nặng: $\mathrm{F0}, \mathrm{F0}-110$, F0-130, F0-150.

Tứ khóa: Cắt thanh quản bán phần, chương trình PRATT, chỉ số Shimmer, Jitter, HNR, thanh điệu, tần số âm cơ bản, biên độ âm cơ bản, trường độ âm.

\section{SUMMARY \\ EVALUATION OF VOICE DISORDERS IN PATIENTS AFTER PARTIAL LARYNGECTOMY}

Partial laryngectomy accounts for $31.2 \%$ of all laryngeal cancer surgeries. The removal of part of the larynx will change the voice. We conducted the study "Evaluation of voice disorders in patients after partial laryngectomy" in a group of 30 patients submitted to partial laryngectomy at the National Hospital of Otorhinolaryngology. Their voices were analyzed before and after partial laryngectomy by the PRAAT acousticvoice analysis system. Results: Age: 45-65

\footnotetext{
${ }^{1}$ Trường Đại Học Y Hà Nội

²Bênh viện Đại học Y Hà Nội

Chịu trách nhiệm chính: Đàm Thu Hiền

Email: hiendamthuent@gmail.com

Ngày nhận bài: 7.5.2021

Ngày phản biện khoa học: 25.6.2021

Ngày duyệt bài: 9.7.2021
}

years old: $63.33 \%$, mean 60.16 , male $100 \%$. Reason for admission: hoarseness: $100 \%$. Voice assessment before partial laryngectomy: Vowel pronunciation: neutral tone (Shimmer index 5.673, Jitter 2.005\%, HNR 26.783), fundamental frequency: $128 \pm 2.8$; basicamplitude: $36 \pm 2.1$, basic field: $89 \pm 3.7$. Tone: Unsigned: F0, F0-30, F0-50, F0-60, grave accent: F0, F0-30, F0-50, F0-80, acute accent: F0- 60, F0-80, F0 +70 , question accent: F0 -F0-85, F0-90, F0-F0+30, fall accent: F0, F0-60, F0+40, F0+90, heavy accent: F0, F0-90, F0-120, F0-140. Voice assessment after partial laryngectomy: neutral tone (Shimmer index 5.874, Jitter 2.452, HNR 28.459; fundamental frequency: $98 \pm 3.7$; fundamental amplitude: $47 \pm$ 4.3, fundamental field: $119 \pm 5.6$. Tone: Unsigned: F0, F0-50, F0-70, F0-80, grave accent: F0, F0-55, F076, F0-90, acute accent: F0-70, F0 -90, F0+60, question accent: F0, F0-95, F0-105, F0-120, F0-90, F0-80, F0+30, fall accent: F0, F0-70, FO+30, FO +50, heavy accent: F0, F0 - 110, F0-130, F0-150.

Keywords; Partial laryngectomy, PRATT program, Shimmer index, Jitter, HNR, tone, fundamental frequency, fundamental amplitude, sound field.

\section{I. ĐẶT VẤN ĐỀ}

Ung thư thanh quản (UTTQ) là bệnh lý có số lượng bệnh nhân đứng thứ 2 trong số ung thư đầu mặt cổ (sau ung thư vòm) ${ }^{1}$. Năm 2016, tại Mỹ có 13.430 trường hợp mắc mới, 3.620 người tử vong vì ung thư thanh quản². Bệnh hay gặp ở nam giới 40- 70 tuổi. Thuốc lá và rượu được coi là yếu tố nguy cơ hàng đầu gây bệnh. Phẫu thuật là phương pháp điều trị chủ yếu cho ung thư thanh quản ${ }^{3,4}$. Việc chẩn đoán sớm giai đoạn của ung thư thanh quản đóng vai trò chính, quyết định phương pháp phẫu thuật tối ưu cho bệnh nhân nhằm mục tiêu chính là kiểm soát tốt khối u, đồng thời bảo tồn chức năng phát âm của thanh quản. Phẫu thuật cắt thanh quản bán phần tuy vẫn đảm bảo chức năng phát âm nhưng ảnh hưởng tới chất lượng giọng sau phẫu thuậat ${ }^{5}$. Nhiều người bệnh khi được bác sỹ tư vấn về Iợi ích của phẫu thuật và di chứng về giọng sau phẫu thuật đã rất lo lắng về chất lượng giọng của họ và trì hoãn việc phẫu thuật, dẫn đển giai đoạn của UTTQ không còn như lúc khám và chỉ định phẫu thuật ${ }^{6}$. Việc đánh giá sự thay đổi giọng nói của bệnh nhâu sau cắt TQBPP là rất cần thiết để cung cấp cho các bác sỹ phẫu thuật có số liệu để tư vấn cho người bệnh để phẫu thuật sớm, đồng thời có kế hoạch phối hợp với các chuyên gia phục hồi giọng nói cho người 
bệnh sớm sau phẫu thuật, giúp người bệnh tự tin có thể có được giong nói tốt. Chính vì những lý do đó, chúng tôi tiến hành nghiên cứu đề tài: "Đánh giá rối loạn giong của bênh nhân sau cắt thanh quản bán phần" với mục tiêu: Đánh giá rối loạn giong của bệnh nhân sau cắt thanh quản bán phần.

\section{II. ĐỐI TƯợNG VÀ PHƯƠNG PHÁP NGHIÊN CỨU}

2.1. Đối tượng nghiên cứu. 30 bệnh nhân ung thư thanh quản được phẫu thuật cắt TQBP tại bệnh viện Tai Mũi Họng Trung Ương, được phân tích giong trước và sau phẫu thuật cắt TQBP bằng chương trình phân tích âm PRAAT.

\subsubsection{Tiêu chuân lựa chọn bệnh nhân}

- Các bênh nhân được chẩn đoán là UTTQ được phẫu thuật cắt TQBP theo Protocol mẫu có đầy đủ hồ sơ bệnh án.

- Không xạ trị trước phẫu thuật

- Được ghi âm giọng nói trước và sau phẫu thuật ít nhất 1 tháng.

2.1.2.Tiêu chuẩn loại trừ. Hỏng mẫu câu ghi âm trong quá trình phần tích

2.2. Phương pháp nghiên cứu: Nghiên cứu mô tả từng ca.

2.3. Chọn mâ̂u: Mẫu thuận tiện

2.4. Thư thập các thông số nghiên cứu

Các thông sổ về đặc điểm chung,triệu chứng của đối tượng nghiên cứu, kết quả phân tích giọng trước và sau phẫu thuật cắt TQBP.

\subsection{Các bước tiến hành}

Bước 1: Xây dựng bệnh án mẫu

Bước 2: Thu thập số liệu nghiên cứu

Bước 3: Phân tích số liệu, viết báo cáo kết quả và bàn luận kết quả thu được

Bước 4: Đưa ra kết luận và kiến nghị dựa trên kết quả thu được

2. 6. Phân tích số liệu cụ thể: Các số liệu thu được qua bệnh án nghiên cứu, được xử lý trên phần mềm thống kê $Y$ học SPSS 16.0.

2.7. Đạo đức nghiên cứu. Nghiên cứu này tiến hành khi có sự đồng ý tham gia của người bệnh. Nghiên cứu không ảnh hưởng đến sức khỏe người bệnh, giúp người bệnh được tư vấn về bệnh và những thay đổi về giọng sau phẫu thuật. Đảm bảo tính trung thực, khách quan trong nghiên cứu.

\section{KẾT QUẢ NGHIÊN CỨU}

3.1. Đặc điểm chung của nhóm nghiên cứu

3.1.1. Giới Nam $100 \%$.

3.1.2. Lý do vào viện: $100 \%$ bệnh nhân vào viện vì khàn tiếng.

\subsubsection{Tuổi}

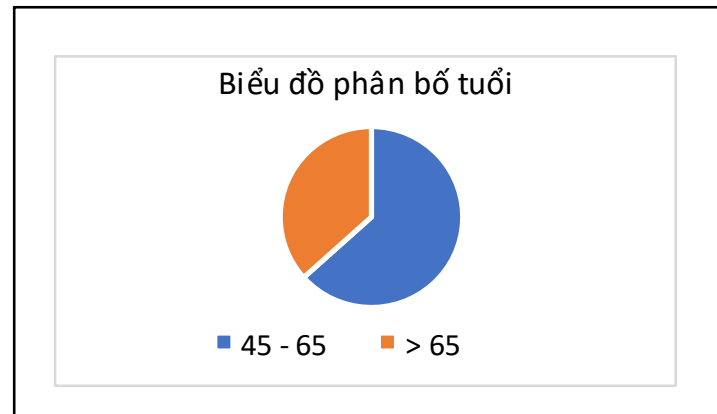

Biểu đồ 3.1:Biểu đồ phân bố tuối.

Nhận xét: Tuổi: 45-65 tuổi: $63.33 \%$. Tuổi trung bình 60.16.

3.2. Phân tích giọng trước và sau cắt thanh quản bán phần

3.2.1. Các chí số nguyên âm

Bảng 3.1. Các chỉ số của nguyên âm ở bênh nhân trước và sau cắt TQBP

\begin{tabular}{|c|c|c|c|}
\hline \multirow{2}{*}{} & \multicolumn{3}{|c|}{$\begin{array}{c}\text { Các chỉ số âm phát âm } \\
\text { nguyên âm trung tính }\end{array}$} \\
\cline { 2 - 4 } & Shimmer & Jitter \% & HNR \\
\hline $\begin{array}{c}\text { Trước cắt } \\
\text { TQBP }\end{array}$ & 5.673 & 2.005 & 26.783 \\
\hline $\begin{array}{c}\text { Sau cắt } \\
\text { TQBP }\end{array}$ & 5.874 & 2.452 & 28.459 \\
\hline
\end{tabular}

Nhân xét: Trước khi cắt TQBP: âm trung tính (chỉ số Shimmer 5.673, Jitter 2.005\%, HNR 26.783), sau cắt TQBP: (chỉ số Shimmer 5.874, Jitter 2.452, HNR 28.459).

3.2.2. Các chỉ số âm cơ bản

Bảng 3.2. Các chỉ số âm cơ bản của bênh nhân trước và sau cắt TQBP

\begin{tabular}{|c|c|c|c|}
\hline & \multicolumn{3}{|c|}{ Các chỉ số âm học } \\
\hline & $\begin{array}{c}\text { Tân số } \\
\text { âm cớ } \\
\text { bản }\end{array}$ & $\begin{array}{c}\text { Biên độ } \\
\text { âm cơ } \\
\text { bản }\end{array}$ & $\begin{array}{l}\text { Trường } \\
\text { độ âm } \\
\text { cở bản }\end{array}$ \\
\hline $\begin{array}{c}\text { Trước cắt } \\
\text { TQBP }\end{array}$ & $\begin{array}{c}128 \pm \\
2.8\end{array}$ & $36 \pm 2.1$ & $89 \pm 3.7$ \\
\hline $\begin{array}{c}\text { Sau cắt } \\
\text { TQBP }\end{array}$ & $98 \pm 3.7$ & $47 \pm 4.3$ & $\begin{array}{c}119 \pm \\
5.6\end{array}$ \\
\hline
\end{tabular}

Nhân xét: Các chỉ số âm học trước khi cắt TQBP: tần số âm cơ bản $128 \pm 2.8$, biên độ âm cơ bản $36 \pm 2.1$, trường độ âm cơ bản $89 \pm 3.7$; sau khi cắt TQBP: tần số âm cơ bản $98 \pm 3.7$, biên độ âm cơ bản $47 \pm 4.3$, trường độ âm cơ bản $119 \pm 5.6$.

3.2.2. Các chỉ sốâmthanh điệu

Bảng 3.3. Các chỉ số âm thânh điệu của bênh nhân trước và sau cắt TQBP

\begin{tabular}{|c|c|c|}
\hline & Trước cắt TQBP & Sau cắt TQBP \\
\hline Thanh & $F 0$, F0-30, F0-50, & F0, F0-50, F0-70, \\
ngang & F0-60 & F0-80 \\
\hline Thanh & F0, F0-30, F0-50, & F0, F0-55, F0-76, \\
huyên & F0-80 & F0-90 \\
\hline Thanh & F0- 60, F0-80, & F0- 70, F0-90, \\
\hline
\end{tabular}




\begin{tabular}{|c|c|c|}
\hline sắc & $\mathrm{F} 0+70$ & $\mathrm{~F} 0+60$ \\
\hline $\begin{array}{l}\text { Thanh } \\
\text { hỏi }\end{array}$ & $\begin{array}{c}\text { F0, F0-85, F0-90, } \\
\text { F0-F0+30 }\end{array}$ & $\begin{array}{c}\text { F0, F0-95, F0- } \\
105, F 0-120, F 0- \\
90, F 0-80, F 0+30\end{array}$ \\
\hline $\begin{array}{c}\text { Thanh } \\
\text { ngã }\end{array}$ & $\begin{array}{c}\mathrm{F} 0, \mathrm{~F} 0-60, \mathrm{FO}+40, \\
\mathrm{~F} 0+90\end{array}$ & $\begin{array}{c}\mathrm{F} 0, \mathrm{~F} 0-70, \mathrm{FO}+30 \\
\mathrm{~F} 0+50\end{array}$ \\
\hline $\begin{array}{l}\text { Thanh } \\
\text { nặng }\end{array}$ & $\begin{array}{c}\text { F0, F0-90, F0- } \\
120, \text { F0-140 }\end{array}$ & $\begin{array}{c}\mathrm{F} 0, \mathrm{~F} 0-110, \mathrm{~F} 0- \\
130, \mathrm{~F} 0-150\end{array}$ \\
\hline
\end{tabular}

Nhận xét: Thanh điệu trước cắt TQBP: Thanh ngang: F0, F0-30, F0-50, F0-60, thanh huyền: $\mathrm{F0}, \mathrm{F0}-30, \mathrm{F0}-50, \mathrm{F0}-80$, thanh sắc: $\mathrm{FO}-$ 60 , F0-80, F0+70, thanh hỏ: F0 -F0-85, F0-90, $F 0-F 0+30$, thanh ngã: $F 0, F 0-60, F 0+40, F 0+90$, thanh nặng: F0, F0-90, F0-120, F0-140.Sau cắt TQBP: Thanh ngang: F0, F0-50, F0-70, F0-80, thanh huyền: $F 0, F 0-55$, F0-76, F0-90, thanh sắc: $F 0-70, F 0-90, F 0+60$, thanh hỏi: $F 0, F 0-95$, $\mathrm{F} 0-105$, F0-120, F0-90, F0-80, F0+30, thanh ngã: $F 0, F 0-70, F 0+30, F 0+50$, thanh nặng: $F 0$, FO - 110, F0-130, F0-150.

\section{BÀN LUẬN}

Đặc điểm chung của bệnh nhân UTTQ. Qua đánh giá 30 bệnh nhân UTTQ đã được phẩu thuật cắt TQBP chúng tôi nhận thây UTTQ hay gặp nhất ở độ tuổi 45-65 với tỷ lệ 63.33\%, tuổi trung bình là 60.16. kết quả này cũng phù hợp với nghiên cứu của Đoàn Hồng Nhật $(2018)^{5}$ với tỷ lệ ung thư thanh quản gặp chủ yếu ở độ tuổi $41-60$ chiếm $73.8 \%$, nghiên cứu của Di Nicola $(2006)^{6}$ với tuổi trung bình là 58.8 , chủ yếu trong khoảng 48-70.100\% bệnh nhân UTTQ là nam giới. Kết quả này cũng phù hợp với nghiên cứu của Quản Thành Nam (2013) ${ }^{7}$, Di Nicola $(2006)^{6}$ Gökhan Demir 20168. Đặc điểm UTTQ thường gặp ở nam giới tuổi trung niên cho thây có lẽ ở đổi tượng này có các yếu tố nguy cơ như hút thuốc lá, uống rượu bia, trào ngược họng thanh quản. $100 \%$ bệnh nhân cắt TQBP vào viện vì khàn tiếng. Kết quả này cũng phù hợp với nghiên cứu của Phạm Văn Hữu (2008) ${ }^{9}$. Ung thư thanh quảncó chỉ định cắt TQBP thường là giai đoạn sớm, bệnh nhân thường có dấu hiệu sớm là khàn tiếng và là lý do họ đi khám. Các triệu chứng khác như khó thở, nuốt vướng, nuốt đau... xuất hiện muộn khi khối u đã phát triển gây bít tắc thanh môn, lan lên thượng thanh môn hay hạ họng. Khi đó không còn chỉ định cắt thanh quản bảo tồn nữa.

Phân tích giọng trước và sau cắt thanh quản bán phần

- Phát âm nguyên âm trước khi cắt TQBP: âm trung tính (chỉ số Shimmer 5.673, Jitter 2.005\%, HNR 26.783), sau khi cắt TQBP: âm trung tính (chỉ số Shimmer 5.874, Jitter 2.452, HNR 28.459). Như vậy ta thấy các chỉ số đánh giá mức độ nhiễu loạn của tần số, biên độ và tỷ lệ chất thanh/tiếng ôn đều tăng lên sau phẩu thuật.

- Các chỉ số âm học trước khi cắt TQBP: tần số âm cơ bản $128 \pm 2.8$, biên độ âm cơ bản 36 \pm 2.1 , trường độ âm cơ bản $89 \pm 3.7$; sau khi cắt TQBP: tần số âm cơ bản $98 \pm 3.7$, biên độ âm cơ bản $47 \pm 4.3$, trường độ âm cơ bản $119 \pm$ 5.6. Như vậy, sau cắt TQBP thì tần số âm cơ bản giảm, biên độ âm và trường độ âm cơ bản đều tăng.

Kết quả này cũng phù hợp với nghiên cứu của Di Nicola $2006^{6}$ với các chỉ số Shimmer 20.009, jitter 11.157, NHR 0.635 đều tăng lên sau phẫu thuật., tỷ lệ tiếng ồn cao. Nghiên cứu của Gökhan Demir ${ }^{8}$, cho thấy có sự giảm đáng kể chất lượng giọng nói sau phẫu thuật. Có thể giải thích điêu này do thanh quản sau phẫu thuật đã mất đi cấu trúc thanh môn, mất đi lớp niêm mạc dây thanh vốn đóng vai trò quan trọng trong cơ chế tạo thanh. Sự rung động từ niêm mạc các cấu trúc còn lại không thường xuyên, liên tục và nhịp nhàng như niêm mạc dây thanh bình thường dấn đến âm thanh được tạo ra luôn có phần thô ráp. Sự khép không kín của ống thanh quản mới trong quá trình phát âm làm rò rỉ không khí ra ngoài gây ra hiện tượng nói mệt, hưt hơi ở những bênh nhân này. Tuy nhiên về cơ bản người nghe vấn có thể hiểu được lời nói của bệnh nhân. Điều này rất quan trọng đối với giao tiếp và đời sống xã hội6.

- Thanh điệu trước cắt TQBP: Thanh ngang: $F 0$, F0-30, F0-50, F0-60, thanh huyền: $F 0, F 0$ 30 , F0-50, F0-80, thanh sắc: $\mathrm{F} 0-60, \mathrm{~F} 0-80$, $\mathrm{F} 0+70$, thanh hỏi: F0 -F0-85, F0-90, F0-F0+30, thanh ngã: F0, F0-60, F0+40, F0+90, thanh nặng: F0, F0-90, F0-120, F0-140.

Sau cắt TQBP: Thanh ngang: F0, F0-50, F070 , F0-80, thanh huyền: F0, F0-55, F0-76, FO90, thanh sắc: $\mathrm{F0}-70, \mathrm{F0}-90, \mathrm{~F} 0+60$, thanh hỏi: F0, F0-95, F0-105, F0-120, F0-90, F0-80, F0+30, thanh ngã: $F 0, F 0-70, F 0+30, F 0+50$, thanh nặng: F0, F0 - 110, F0-130, F0-150.

\section{KẾT LUÂN}

Sau cắt TQBP giọng nói bệnh nhân bị thay đổi nhiều do cấu trúc thanh quản đã bị thay đổi, tuy nhiên vẫn có thể giao tiếp hiểu được.

\section{TÀI LIÊUU THAM KHẢO}

1. Ngổ Ngoc Liễn. Ung thư thanh quản. In: Giản Yểu Tai Mũi Hong. Nhà xuất bản Y hoc; 2006:345-348.

2. Forastiere $A A$, Ismaila $\mathbf{N}$, Lewin $\mathbf{J S}$, et al. Use of Larynx-Preservation Strategies in the Treatment of Laryngeal Cancer: American Society of Clinical 
Oncology Clinical Practice Guideline Update. J Clin Oncol. doi:10.1200/JCO.2017.75.7385 2018;36(11):1143-1169.

3. Steuer CE, El-Deiry M, Parks JR, Higgins KA, Saba NF. An update on larynx cancer. CA: A Cancer Journal for Clinicians. 2017;67(1):31-50. doi: $10.3322 /$ caac. 21386

4. Jenckel F, Knecht R. State of the Art in the Treatment of Laryngeal Cancer. Anticancer Res. 2013:33(11):4701-4710.

5. Đoàn Thị Hồng Nhật, Lê Minh Kỳ. Kết quả phẫu thuật cắt thanh quản bán phần trên nhẫn tạo hình kiểu Tucker. Published online 2018.

6. Di Nicola V, Fiorella M, Spinelli D, Fiorella $R$. Acoustic analysis of voice in patients treated by reconstructive subtotal laryngectomy. Evaluation and critical review. Acta Otorhinolaryngol Ital. 2006;26(2):59-68.

7. Quản Thanh Nam, Lê Minh Kỳ. Đánh giá kết quả phẫu thuât cắt thanh quản bán phần kiểu Tucker trong điều tri ung thư thanh quản giai đoan T1b, T2 tại bệnh viện Tai Mũi Họng Trung Ương. Published online 2013.

8. Gökhan Demir M, Paksoy M, Sanlı A, et al. Subjective and objective evaluation of voice and pulmonary function in partial laryngectomised patients. Integr Cancer Sci Therap. 2016;3(1). doi:10.15761/ICST.1000168

9. Phạm Văn Hữu. Nghiên cứu hình thái lâm sàng, nội soi đối chiếu với kết quả phẫu thuật của ung thư thanh quản giai đoạn sớm. Published online 2008.

\section{ĐĂC ĐIỂM BÊNH LÝ VÀ ỨNG DỤNG KỸ THUÂT PHẪU THUÂ̂T NộI SOI HOÀN TOẦN NGOÀI PHÚC MAC (TEP) ĐĂT LƯỚI NHÂN TAOO 3D ĐIỀU TRI THOÁT Vİ BẸN HAI BÊN Ở NGƯỜI LỚN}

\section{TÓM TẮT}

Mục tiêu: Mô tả đặc điểm bệnh lý và ứng dụng kỹ thuật phẩu thuật nội soi hoàn toàn ngoài phúc mac (Total Extraperitoneal - TEP) đặt lưới nhân tạo 3D điêu trị thoát vị bẹn hai bên ở người lớn. Đối tượng và phương pháp: Nghiên cứu mô tả 60 bệnh nhân (BN) trên 18 tuổi được chẩn đoán là thoát vị bẹn (TVB) hai bên và được điều trị bằng phẫu thuật nội soi TEP đă̆t lưới nhân tạo 3D tại Bệnh viện Thanh Nhàn, từ tháng 01/2017 đến tháng 11/2020. Kết quả: $100 \%$ BN nam, tuổi trung bình 51,0. TVB trực tiếp chiếm đa số với $63,3 \%$. Phân loaai theo Nyhus loại I $3,3 \%$, loại II $26,7 \%$, loại IIIA $63,3 \%$ và IIIB là $6,7 \%$. $91,7 \%$ phấu tích táo khoang trước phúc mạc (PM) bằng ngón tay và khí $\mathrm{CO}_{2} .63,3 \%$ túi thoát vị được đẩy vào trong ổ bung. $80,0 \%$ sử dụng lưới $3 \mathrm{D}$ loại nhỏ, 1 trường hợp $(1,7 \%)$ phải khâu cố định lưới. Tai biến $15 \% .1,7 \%$ thêm 1 trocar. Thời gian đặt lưới trung bình 21,1 phút, thời gian phẫu thuật trung bình 74,9 phút. Kết luận: PTNS hoàn toàn ngoài phúc mạc đặt lưới nhân tạo 3D là phương pháp khả thi, an toàn trong điều trị thoát vị bẹn hai bên ở người lớn.

\section{SUMMARY \\ PATHOLOGICAL CHARACTERISTICS AND APPLICATION OF LAPAROSCOPIC TOTALLY}

\footnotetext{
*Bênh viện Thanh Nhàn

**Bộ môn ngoại - ĐHYD Hải Phòng

***Trung tâm PTTH - BVTW 108

Chịu trách nhiệm chính: Nguyễn Văn Phước

Email: drphuocbvtn@gmail.com

Ngày nhận bài: 10.5.2021

Ngày phản biện khoa học: 28.6.2021

Ngày duyệt bài: 12.7.2021
}

Nguyễn Văn Phước*, Hà Văn Quyết**, Đào Quang Minh*, Vũ Ngọc Sơn***

EXTRAPERITONEAL USING 3D MESH TO TREAT BILATERAL INGUINAL HERNIA IN ADULTS

Objective: This study aimed to describe pathological characteristics and application of laparoscopic totally extraperitoneal (TEP) using 3D mesh to treat bilateral inguinal hernia in adults. Methods: Descriptive study 60 patients with bilateral inguinal hernias undergoing laparoscopic TEP surgery using 3D mesh at Thanh Nhan Hospital from January 2017 to November 2020. Results: All the patients were male, mean age were 51.0 years. Direct hernias were more commons comprising of $63.3 \%$. Classification according to Nyhus type I 3.3\%, type II $26.7 \%$, class IIIA $63.3 \%$ and IIIB $6.7 \%$. $91.7 \%$ preperitoneal cavity were dissection with finger and CO2. $63.3 \%$ of the hernia sac was pushed into the abdomen. The small 3D mesh was used in most case $(80 \%), 1$ case $(1,7 \%)$ was stitched fixation mesh. Intraoperative complications were $15 \% .1 .7 \%$ must be additional trocar. The average mesh put time was 21.1 minutes. The mean operative time was 74.9 minutes. Conclusion: TEP laparoscopic surgery using $3 \mathrm{D}$ mesh is a safe, feasible, and effective method in bilateral inguinal hernia in adults.

\section{I. ĐẶT VẤN ĐỀ}

TVB là tình trang bệnh lý phổ biến xảy ra ở khoảng $1-5 \%$ dân số nói chung, trong đó 15 $20 \%$ là TVB hai bên. Phẫu thuật nội soi (PTNS) điều trị TVB lần đầu được báo cáo bởi Ger năm 1982. Kể từ đó đến nay, bên cạnh những cải tiến về kỹ thuật, sự ra đời và phát triển của lưới nhân tạo cũng tạo ra những thay đổi mang tính cách mạng trong điêu trị TVB. Năm 1999, W. Bell [1] lần đầu tiên sử dụng tấm lưới nhân tạo 3D với 